\title{
A GENERALIZATION OF A THEOREM OF JANOS AND EDELSTEIN
}

\author{
SEHIE PARK
}

\begin{abstract}
A generalization of Edelstein's version of a theorem of Janos and its converse are obtained:

TheOREM. Let $X$ be a compact metrizable topological space, and $f, g$ be continuous self-maps of $X$ such that $g f=f g$ and $f$ is bijective. Then $g$ is injective and $\bigcap_{1}^{\infty} g^{n} X=\left\{x_{0}\right\}$, where $x_{0} \in X$, iff, given $\lambda, 0<\lambda<1, a$ homeomorphism $h$ of $X$ into $l_{2}$ exists such that

$$
\|h(g x)-h(g y)\|=\lambda\|h(f x)-h(f y)\|
$$

for all $x, y \in X$.
\end{abstract}

In [2], [3] and [4], Janos obtained the following

THEOREM (JANOS). Let $X$ be a compact metrizable topological space and $f$ : $X \rightarrow X$ a continuous one-to-one mapping with $\cap_{1}^{\infty} f^{n}[X]$ a singleton. Given $\lambda$, $0<\lambda<1$, there exists a metric $\rho$ on $X$ such that the metric topology of $(X, \rho)$ is identical with the original one and $\rho(f x, f y)=\lambda \rho(x, y)$ for all $x, y \in X$.

M. Edelstein [1] gave a somewhat stronger result as follows:

THEOREM (EDELSTEIN). Let $X$ be a compact metrizable topological space and $f: X \rightarrow X$ a continuous one-to-one mapping with $\cap_{1}^{\infty} f^{n}[X]=\left\{x_{0}\right\}$, where $x_{0} \in X$. Given $\lambda, 0<\lambda<1$, a homeomorphism $h$ of $X$ into $l_{2}$ exists such that

$$
\left\|h\left(f x^{\prime}\right)-h\left(f x^{\prime \prime}\right)\right\|=\lambda\left\|h x^{\prime}-h x^{\prime \prime}\right\| \text { for all } x^{\prime}, x^{\prime \prime} \in X \text {. }
$$

Note that the converse of Edelstein's theorem holds by the Banach contraction principle.

In this paper we show that a more general result than Edelstein's holds. We need the following result from [5].

THEOREM (JUNGCK). Let $f$ be a continuous map of a complete metric space $(X, d)$ into itself. Then $f$ has a fixed point in $X$ iff there exist $\alpha \in(0,1)$ and $a$ map $g: X \rightarrow X$ which commutes with $f$ and satisfies $g X \subset f X$ and $d(g x, g y) \leqslant$ $\alpha d(f x, f y)$ for all $x, y \in X$. Indeed, $f$ and $g$ have a unique common fixed point.

Actually, in case $f=1_{X}$, the identity map of $X$, Jungck's theorem implies the Banach principle. Motivated by this fact, we generalize Edelstein's theorem as follows:

THEOREM. Let $X$ be a compact metrizable topological space, and $f, g$ be

Received by the editors September 27, 1976.

AMS (MOS) subject classifications (1970). Primary 54E40, 54E45, 55C20; Secondary $47 \mathrm{H} 10$.

( ) American Mathematical Society 1977 
continuous self-maps of $X$ such that $g f=f g$ and $f$ is bijective. Then $g$ is injective and $\cap_{1}^{\infty} g^{n} X=\left\{x_{0}\right\}$, where $x_{0} \in X$, iff, given $\lambda, 0<\lambda<1$, a homeomorphism $h$ of $X$ into $l_{2}$ exists such that

$$
\|h(g x)-h(g y)\|=\lambda\|h(f x)-h(f y)\| \quad \text { for all } x, y \in X .
$$

Proof. Necessity. We may assume $X \backslash\left\{x_{0}\right\} \neq \varnothing$. Let $\mathscr{B}$ be a countable base for the open nonempty set $X \backslash g X$. For each pair $U, V \in \mathscr{B}$, such that $\bar{U} \subset V$, there is a continuous $\phi: X \rightarrow[0,1]$ such that $\phi U=1$ and $\phi(X \backslash V)$ $=0$. Using the odd positive integers as an index set we obtain a family $\left\{\phi_{2 n-1} \mid n=1,2, \ldots\right\}$ of maps. Since $g X$ is closed in $X$ and $\phi_{2 n-1} f g^{-1}$ : $g X \rightarrow[0,1]$ is continuous for each $n=1,2, \ldots$, there exists, by the Tietze extension theorem, a continuous $\phi_{2(2 n-1)}: X \rightarrow[0,1]$ which coincides with $\phi_{2 n-1} f g^{-1}$ on $g X$. Thus $\phi_{2(2 n-1)}(g x)=\phi_{2 n-1}(f x)(n=1,2, \ldots ; x \in X)$. Assuming $\phi_{2^{m-1}(2 n-1)}: X \rightarrow[0,1]$ defined, continuous and satisfying

$$
\phi_{2^{m-1}(2 n-1)}(g x)=\phi_{2^{m-2}(2 n-1)}(f x) \quad(n=1,2, \ldots ; x \in X),
$$

we define $\phi_{2^{m}(2 n-1)}: X \rightarrow[0,1]$ by choosing a continuous extension of $\phi_{2^{m-1}(2 n-1)} f g^{-1}: g X \rightarrow[0,1]$ to $X$, thereby obtaining continuous maps $\phi_{2^{m}(2 n-1)}: X \rightarrow[0,1]$ for all $m=1,2, \ldots ; n=1,2, \ldots$ and satisfying

$$
\phi_{2^{m}(2 n-1)}(g x)=\phi_{2^{m-1}(2 n-1)}(f x) \quad(m=1,2, \ldots ; n=1,2, \ldots ; x \in X)
$$

and $\phi_{2 n-1}(g x)=0(n=1,2, \ldots ; x \in X)$.

We now define $h$ as follows: If $k=2^{m}(2 n-1)$ we set

$$
z_{k}=\lambda^{m+n} \phi_{2^{m}(2 n-1)}(x) \text { and } h x=\left(z_{1}, z_{2}, \ldots, z_{k}, \ldots\right) .
$$

Obviously $h x \in l_{2}$. It is easy to see that $h$ is injective and continuous, hence, by the compactness of $X$, a homeomorphism onto $h X$. Finally,

$$
\begin{aligned}
\|h(g x)-h(g y)\|^{2} & =\sum_{m=0}^{\infty} \sum_{n=1}^{\infty} \lambda^{2(m+n)}\left[\phi_{2^{m(2 n-1)}}(g x)-\phi_{2^{m(2 n-1)}}(g y)\right]^{2} \\
& =\sum_{m=1}^{\infty} \sum_{n=1}^{\infty} \lambda^{2(m+n)}\left[\phi_{2^{m-1}(2 n-1)}(f x)-\phi_{2^{m-1}(2 n-1)}(f y)\right]^{2} \\
& =\lambda^{2} \sum_{m=0}^{\infty} \sum_{n=1}^{\infty} \lambda^{2(m+n)}\left[\phi_{2^{m(2 n-1)}}(f x)-\phi_{2^{m(2 n-1)}}(f y)\right]^{2} \\
& =\lambda^{2}\|h(f x)-h(f y)\|^{2} .
\end{aligned}
$$

Sufficiency. Since $X$ can be identified with $h X$, there exist continuous maps $\bar{f}, \bar{g}: h X \rightarrow h X$ such that $h f=\bar{f} h$ and $h g=\bar{g} h$. Then we have

$$
\|\bar{g}(h x)-\bar{g}(h y)\|=\lambda\|\bar{f}(h x)-\bar{f}(h y)\|
$$

for all $x, y \in X$. Hence, there is a metric $d$ on $X$ such that $d(g x, g y)=$ $\lambda d(f x, f y)$ for all $x, y \in X$. This shows that $g$ is injective and that $f$ and $g$ have a unique common fixed point $x_{0}$ by Jungck's theorem. Since $X$ is bounded, for any $\varepsilon>0$ there is an $N>0$ such that $n>N$ implies $\lambda^{n}$ diam $X<\varepsilon$. Then 


$$
\begin{aligned}
d\left(g^{n} x, x_{0}\right) & =d\left(g^{n} x, g^{n} x_{0}\right)=\lambda^{n} d\left(f^{n} x, f^{n} x_{0}\right) \\
& =\lambda^{n} d\left(f^{n} x, x_{0}\right)<\lambda^{n} \operatorname{diam} X<\varepsilon,
\end{aligned}
$$

whence we have $\cap_{1}^{\infty} g^{n} X=\left\{x_{0}\right\}$.

REMARK. The necessity part of the proof is essentially due to Edelstein. In case $f=1_{X}$, our theorem reduces to Edelstein's.

\section{REFERENCES}

1. M. Edelstein, A short proof of a theorem of L. Janos, Proc. Amer. Math. Soc. 20 (1969), 509-510. MR 38 \#2743.

2. L. Janos, Converse of the Banach theorem in the case of one-to-one contracting mapping, Notices Amer. Math. Soc. 11 (1964), 686. Abstract \#64T-469.

3. , Homothetic property of contractive one-to-one mappings, Notices Amer. Math. Soc. 13 (1966), 818. Abstract \#638-11.

4. One-to-one contractive mappings on compact space, Notices Amer. Math. Soc. 14 (1967), 133. Abstract \#67T-21.

5. G. Jungck, Commuting mappings and fixed points, Amer. Math. Monthly 83 (1976), 261-263.

Mathematics Department, Seoul National Universtty, Seoul 151, Korea 